\title{
Implementación de un modelo de atención kinesiológico, temprano y prospectivo, en personas con cáncer de mama
}

\author{
Karol Ramírez-Parada ${ }^{1}$, Williams Mella-Abarca ${ }^{1}$, Natalia Nicoletti-Santoni ${ }^{1}$, \\ Gustavo Torres-Riveros ${ }^{1}$, Ignacio Villagrán-Gutiérrez ${ }^{1}$, \\ Militza Petric-Guajardo y Raúl Aguilera-Eguía ${ }^{3}$
}

${ }^{1}$ Carrera de Kinesiología. Departamento de Ciencias de la Salud. Facultad de Medicina. Pontificia Universidad Católica de Chile. Santiago, Chile.

${ }^{2}$ Complejo Asistencial Dr. Sótero del Río. Santiago

${ }^{3}$ Departamento de Salud Pública. Facultad de Medicina

Kinesiología. Universidad

Católica de la Santísima

Concepción. Concepción

Recibido el 18 de julio de 2019 y aceptado para publicación el 30 de julio de

Correspondencia a: Karol Ramírez-Parada kramirezp@uc.cl

Implementation of an early and prospective physical therapy model care in people with breast cancer

Breast cancer is a public health problem in Chile and the world due to high incidence. Surgery is the mainstay in the breast cancer treatment. Up to $60 \%$ of people receiving this management have functional morbidity in the upper limb. There are rehabilitation health models care for the functional morbidity, however, in Chile is not a standard in the health care. This article describes: 1) upper limb morbidity secondary to breast cancer surgery, and 2) early and prospective physical Therapy model care for people with breast cancer.

Key words: breast neoplasm; physical therapy; rehabilitation; upper limb; functionality morbidity.

\section{Resumen}

El cáncer de mama es un problema de salud pública en Chile y el mundo por su alta incidencia. La cirugía es un pilar fundamental en el tratamiento del cáncer de mama. Hasta un $60 \%$ de las personas tratadas por cáncer de mama presentan alteraciones funcionales en el miembro superior. Existen modelos sanitarios para la rehabilitación de las alteraciones funcionales, sin embargo, en Chile no es un estándar en la atención de salud. Este artículo describe: 1) las alteraciones del miembro superior secundarias a la cirugía por cáncer de mama y 2) un modelo de atención kinesiológico, temprano y prospectivo, para personas tratadas por cáncer de mama.

Palabras clave: neoplasia de mama; terapia física; rehabilitación; miembro superior; alteraciones funcionales.

\section{Introducción}

El cáncer de mama $(\mathrm{CM})$ es el cáncer más frecuente y la principal causa de muerte por cáncer en mujeres a nivel mundial ${ }^{1}$. En Chile, la incidencia es de 41 por 100.000 mujeres/año y es la primera causa de muerte por cáncer en mujeres ${ }^{2}$. Por lo tanto, el $\mathrm{CM}$ es un problema de salud pública relevante para Chile.

En el CM temprano, aún no hay diseminación de las células cancerígenas más allá de la mama o de los ganglios linfáticos de la axila e incluye los cánceres de mama en estadios I, II y III ${ }^{3}$. En Chile, alrededor de $95 \%$ de los diagnósticos de $\mathrm{CM}$ se pesquisan en etapa temprana ${ }^{4}$. Esto último, permite que la sobrevida relativa a cinco años sea de $81 \% 5$.

El manejo habitual del CM temprano depende de su biología y puede incluir cirugía, quimioterapia, radioterapia, hormonoterapia e inmunoterapia ${ }^{6}$. $\mathrm{La}$ combinación y avances en estos tratamientos ha sido efectiva en aumentar la sobrevida global de las personas tratadas por $\mathrm{CM}^{7}$. Sin embargo, se estima que un $60 \%$ de ellas presentan alteraciones secundarias a corto y largo plazo ${ }^{8-11}$.

La cirugía es uno de los pilares fundamentales del tratamiento del CM. Este tratamiento se asocia a alteraciones en el miembro superior, tales como disminución del rango articular de movimiento, sín- 
drome de red axilar y linfedema ${ }^{12}$. Estas alteraciones disminuyen la funcionalidad de los miembros superiores y deterioran la calidad de vida de las personas tratadas por $\mathrm{CM}^{12,13}$.

Si bien, existen modelos sanitarios que incluyen rehabilitación en el manejo de enfermedades crónicas, aquellos enfocados a personas con CM son un fenómeno relativamente reciente ${ }^{14}$. Se ha reportado que programas kinesiológicos oportunos, pueden prevenir o resolver las alteraciones en el miembro superior secundarias a la cirugía por $\mathrm{CM}^{1,14,15}$. No obstante, la derivación a profesionales del área de la rehabilitación no es un estándar en la mayoría de los sistemas de salud ${ }^{16}$. En Chile, existen 42 unidades de patología mamaria, y apenas el $25 \%$ reporta que cuentan con kinesiólogos en sus equipos ${ }^{17}$.

Un modelo kinesiológico en CM, temprano y prospectivo, propone realizar una evaluación periódica y continua, incluso en ausencia de deterioro funcional. Por tanto, podría permitir la detección e intervención temprana de alteraciones en el miembro superior ${ }^{14,18-20}$. Los objetivos de este artículo son: describir las alteraciones del miembro superior secundarias a la cirugía por CM y describir un modelo de atención kinesiológico temprano y prospectivo para personas tratadas por CM.

\section{Alteraciones del miembro superior secundarias a la cirugía por cáncer de mama}

Las alteraciones del miembro superior secundarias a la cirugía por $\mathrm{CM}$, se caracterizan por compromiso linfovascular, que generan alteraciones como el síndrome de red axilar y linfedema ${ }^{10,14,21}$. Además, las personas tratadas por CM pueden presentar problemas de movilidad articular del miembro superior asociada a causas mecánicas (musculoesqueléticas, tegumentarias o nerviosas) o por sintomatología asociada al periodo poscirugía ${ }^{21}$. Estas alteraciones, impactan negativamente la función de los miembros superiores y pueden incrementarse por otros tratamientos antineoplásicos, como la radioterapia y/o quimioterapia ${ }^{12,13}$.

El síndrome de red axilar se define como la presencia de uno o varios cordones fibrosos, palpables y visibles, en la región axilar y que pueden extenderse hasta la muñeca ${ }^{22}$. Este síndrome se asocia con dolor y limitación del rango de movimiento del hombro y $\operatorname{codo}^{12,23}$. Mientras que el linfedema, es una condición crónica que se caracteriza por aumento de volumen en el brazo del lado de la cirugía ${ }^{20}$. El linfedema es consecuencia de la ablación linfática y afecta aproximadamente a un tercio de las personas tratadas por $\mathrm{CM}^{12}$

Por otro lado, las alteraciones mecánicas se manifiestan con déficit de la fuerza muscular y del rango articular de los movimientos del hombro, en flexión, rotación externa y abducción ${ }^{12,13,21}$. Cabe señalar, que estos movimientos son fundamentales en varias actividades de la vida diaria, tales como peinarse y alcanzar objetos por sobre el nivel del hombro.

\section{Modelo de atención kinesiológico, temprano y prospectivo, para personas con $\mathrm{CM}$}

Este modelo propone evaluación y educación continua a la persona tratada por CM. De esta evaluación se obtiene un diagnóstico kinesiológico, basado en la clasificación internacional para la funcionalidad (CIF) que complementa el diagnóstico médico $^{24}$. El diagnóstico kinesiológico considera factores biopsicosociales en torno al movimiento humano.

Las personas tratadas por CM pueden presentar alteraciones secundarias a la cirugía. Estas alteraciones se pueden agrupar en tres patrones alterados: 1) preventivo latente; 2) limitación funcional por alteración linfovascular y 3) limitación funcional por alteración mecánica (Tabla 1). La identificación del patrón alterado puede guiar los objetivos y técnicas del tratamiento kinesiológico.

Tabla 1. Patrones kinesiológicos alterados en personas tratadas por cáncer de mama

Patrones
Preventivo latente
$\begin{aligned} & \text { Limitación funcional por alteración linfovascular } \\ & \text { a) Síndrome de red axilar } \\ & \text { b) Linfedema }\end{aligned}$

Limitación funcional por alteración mecánica

\section{Descripción}

Persona sometida a mastectomía parcial o total con cirugía en axila y sin limitación funcional

Persona con presencia de:

a) Cordones linfáticos palpables y/o visibles en la región axilar y que pueden extenderse hasta la muñeca

b) Aumento de volumen en el brazo del lado de la cirugía

Persona con limitación del rango articular de movimiento y/o fuerza muscular del miembro superior 
El modelo de atención kinesiológico, temprano y prospectivo, en CM propone que toda persona con indicación de cirugía por $\mathrm{CM}$ reciba una atención kinesiológica precirugía. Esta atención kinesiológica debe incluir evaluación y educación. Posteriormente, recibe intervención kinesiológica un mes poscirugía. En la intervención kinesiológica poscirugía, se define el patrón alterado y se comienza con el seguimiento prospectivo (Figura 1).

\section{Atención kinesiológica precirugía}

El objetivo de la atención kinesiológica precirugía es determinar parámetros funcionales de la condición física basal y educar sobre las posibles alteraciones del miembro superior secundarias a la cirugía. En esta atención, se propone una entrevista clínica y una batería de pruebas funcionales (Tabla 2).

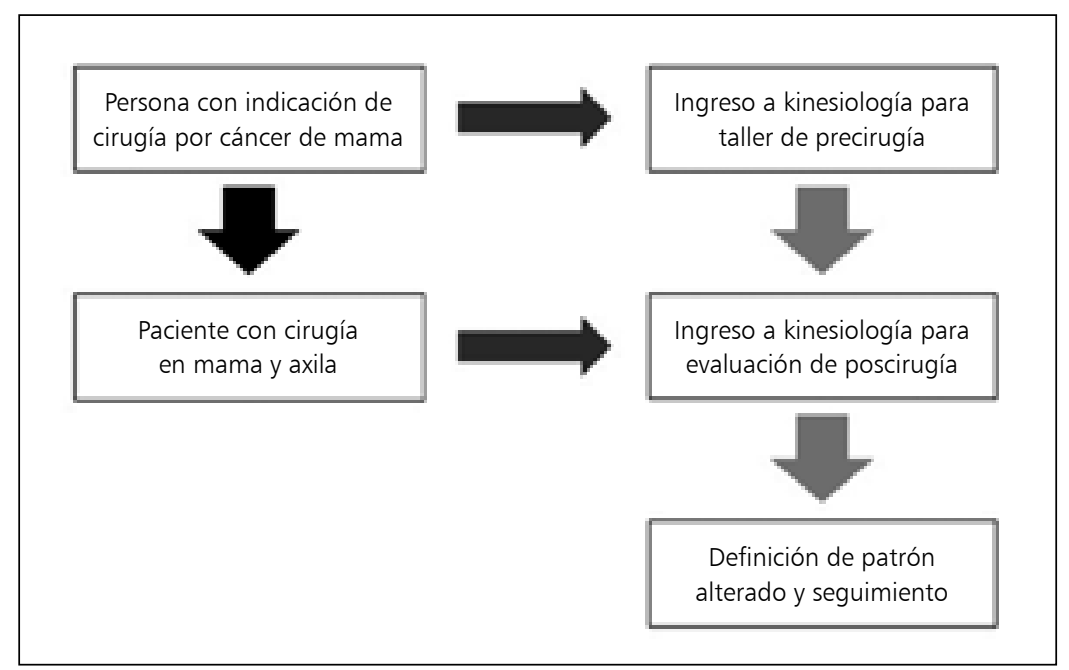

Figura 1. Modelo de atención kinesiológico en cáncer de mama.
La educación se realiza en cuatro ejes temáticos: 1) posibles alteraciones del miembro superior secundarias a la cirugía de la mama y axila; 2) ejercicio físico antes y después de la cirugía; 3) la importancia de la detección y tratamiento precoz de las alteraciones del miembro superior secundarias a la cirugía y 4) cuidados generales del miembro superior.

\section{Atención kinesiológica poscirugía}

El objetivo de esta atención es detectar y tratar los patrones alterados del miembro superior de manera temprana y oportuna. En esta evaluación se aplica la batería de pruebas funcionales (Tabla 2). Además, se incluye: 1) evaluación de presencia del síndrome de red axilar; 2) evaluación traumatológica de cintura escapular. Es importante definir el diagnóstico kinesiológico y su respectivo patrón para definir el seguimiento. El tratamiento específico según patrón se representa en la Tabla 3.

\section{Seguimiento kinesiológico prospectivo}

El objetivo del seguimiento es mantener un adecuado control de la persona tratada por CM según su patrón alterado y evolución. La periodicidad de los controles se define según el patrón alterado (Figura 2).

Las personas con patrón preventivo latente, son aquellos sometidos a mastectomía parcial o total con cirugía en axila. Las personas con biopsia de linfonodo centinela sin otra alteración son evaluados $1 \mathrm{y}$ 6 meses poscirugía. Posterior a esas dos sesiones, se indica alta kinesiológica. En el caso de las personas con disección axilar y/o SRA resuelto son evaluados los meses 1, 2 y 3 poscirugía. Posteriormente, las sesiones son cada tres meses hasta completar un seguimiento de dos años poscirugía. Todas las

Tabla 2. Batería de herramientas de evaluación

\begin{tabular}{|lll|}
\hline Período & Herramienta & Resultado \\
\hline Pre y poscirugía & Prueba de marcha 2 minutos & Capacidad funcional cardiorrespiratoria \\
& Dinamometría & Fuerza muscular de prensión manual \\
& Prueba de Apley & Rango funcional de movimiento de hombros \\
& Cuestionario calidad de vida QLQ-C30 y QLQ-BR23 & Calidad de vida en paciente con cáncer y anexo para CM \\
& $\begin{array}{l}\text { Nomograma de predicción del riesgo de linfedema secun- } \\
\text { dario a CM }\end{array}$ & $\begin{array}{l}\text { Riesgo de desarrollar linfedema a cinco años en una escala } \\
\text { de 0 a 100\% }\end{array}$ \\
& Circometría de los miembros superiores & Medición de perímetros y volumen \\
Poscirugía & Observación y palpación de síndrome de red axilar & Presencia de síndrome de red axilar \\
& Evaluación traumatológica de cintura escapular & $\begin{array}{l}\text { Postura, patrón de movimiento de la cintura escapulo- } \\
\text { humeral y estado de los tejidos blandos }\end{array}$ \\
& &
\end{tabular}


CIRUGía AL díA

Tabla 3. Tratamiento según patrón

\begin{tabular}{|l|l|}
\hline Patrón & Técnicas de tratamiento \\
Preventivo latente & $\begin{array}{l}\text { Educación y ejercicio terapéutico (miolinfokinéticos, de rango articular y fuerza muscular } \\
\text { de miembro superior) }\end{array}$ \\
$\begin{array}{l}\text { Limitación funcional por alteración linfovascular } \\
\begin{array}{l}\text { a) Síndrome de red axilar } \\
\text { b) Linfedema }\end{array}\end{array}$ & $\begin{array}{l}\text { a) Elongación de tejido blando, terapia manual ortopédica, presoterapia secuencial intermi- } \\
\text { tente, drenaje linfático manual, manga de tejido circular, ejercicio terapéutico y educación } \\
\text { Terapia descongestiva compleja, incluyendo el uso de vendajes de baja elasticidad, man- } \\
\text { ga de tejido plano y ejercicios miolinfokinéticos. Además, se pueden incluir técnicas de } \\
\text { linfotaping y ejercicio físico aeróbico y de resistencia }\end{array}$ \\
\hline Limitación funcional por alteración mecánica & \begin{tabular}{l} 
Terapia manual ortopédica, ejercicio terapéutico, calor superficial y electroterapia tipo TENS \\
\hline
\end{tabular}
\end{tabular}

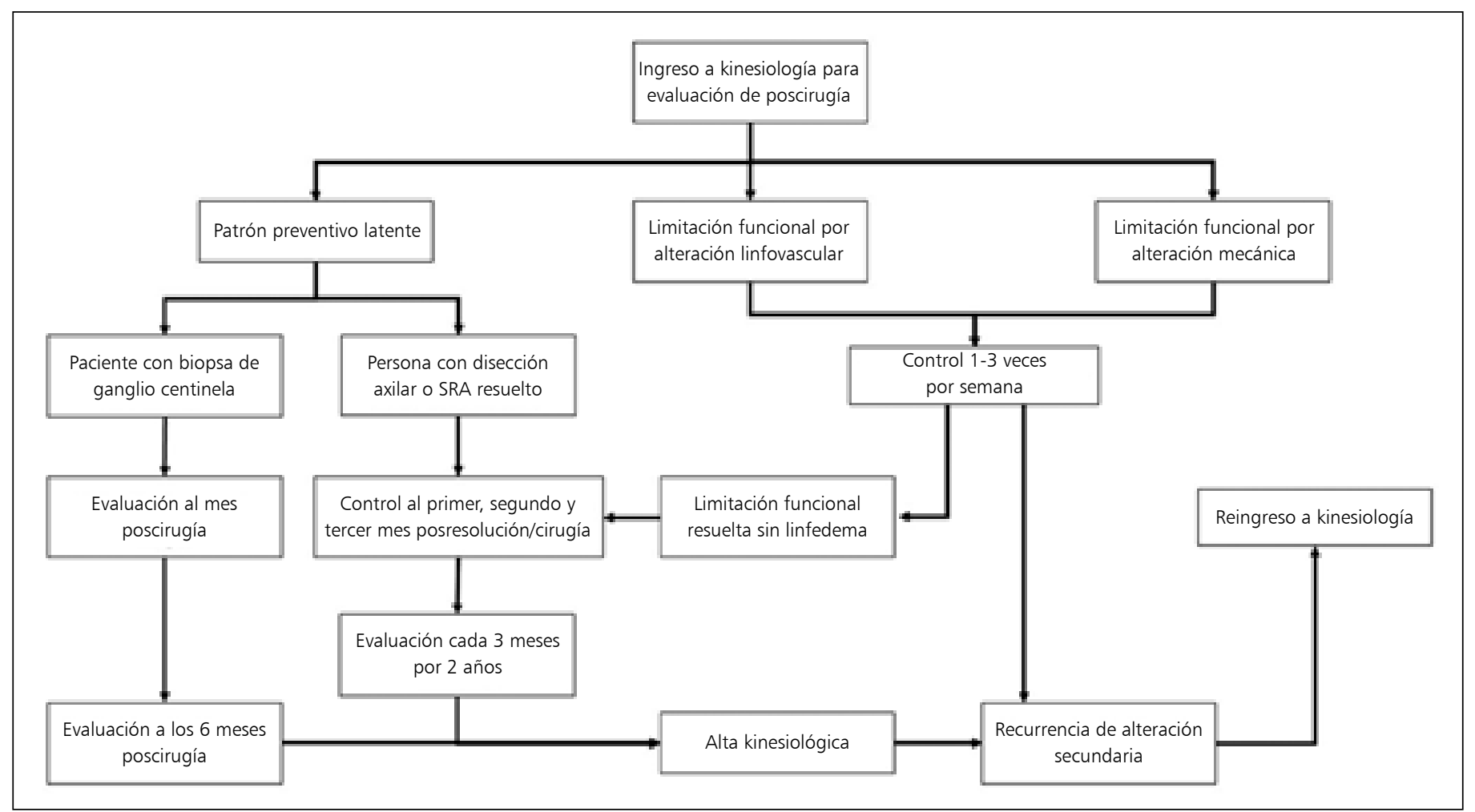

Figura 2. Seguimiento kinesiológico prospectivo.

personas con alta kinesiológica, siempre tendrán la opción de reingresar a kinesiología en caso de presentar alguna alteración del miembro superior secundaria a la cirugía.

Las personas con patrón de limitación funcional por alteración linfovascular o mecánico, se tratarán 1 a 3 veces por semana según la disponibilidad del centro para la realización adecuada de terapia manual ortopédica, ejercicio terapéutico supervisado y terapia descongestiva compleja. Las personas que resuelvan su limitación funcional, y que fueron so- metidas a disección axilar continúan en el protocolo de patrón preventivo.

La educación es fundamental en el modelo de atención temprano y prospectivo. En general, se deben reforzar los cuidados para prevenir alteraciones secundarias, como el linfedema e incentivar estilos de vida saludables. Algunos ejemplos de educación son los siguientes: aumentar el nivel de actividad física, promover la alimentación saludable, consejería antitabaco, cuidados de la piel y ejercicios miolinfokinéticos. 


\section{Conclusión}

El modelo de atención kinesiológico, temprano y prospectivo, con un enfoque preventivo, puede presentar beneficios directos en la atención en salud de las personas tratadas por CM. Sin embargo, estos modelos no se encuentran implementados en las unidades de patología mamaria en Chile. Este artículo puede ser un soporte importante para integrar este modelo de atención. Asimismo, es fundamental determinar el costo-efectividad de este modelo a nivel nacional, lo que significa un desafío importante para los profesionales de la rehabilitación relacionados con el CM en Chile.

\section{Responsabilidades éticas}

Protección de personas y animales. Los autores declaran que para esta investigación no se han realizado experimentos en seres humanos ni en animales.

Confidencialidad de los datos. Los autores declaran que en este artículo no aparecen datos de pacientes.

Conflictos de interés: no hay.

\section{Bibliografía}

1. Bray F, Ferlay J, Soerjomataram I, Siegel RL, Torre LA, Jemal A. Global cancer statistics 2018: GLOBOCAN estimates of incidence and mortality worldwide for 36 cancers in 185 countries. CA Cancer J Clin [Internet]. 2018;68:394-424. Available from: http://www.ncbi.nlm.nih. gov/pubmed/30207593.

2. Globocan 2018. Chile Source: Globocan 2018. 2018;576:1-2.

3. Minsal. Guías Clínicas AUGE Cáncer de Mama. 2015. 164 p.

4. del Castillo C, Cabrera ME, Derio L, Gaete F, Cavada G. Resultados del tratamiento del cáncer de mama, Programa Nacional de Cáncer del Adulto Impact of the Chilean Explicit Guaranties Health System (GES) on breast cancer treatment. Rev Med Chile 2017;145(Globocan 2008):1507-13.

5. Minsal. Plan Nacional de Cáncer 20182028.

6. Curigliano G, Burstein HJ, Winer EP, Gnant M, Dubsky P, Loibl S, et al. Deescalating and escalating treatments for early-stage breast cancer: The St. Gallen International Expert Consensus Conference on the Primary Therapy of Early Breast Cancer 2017. Ann Oncol. 2017;28:1700-12.

7. Narod SA, Iqbal J, Miller AB. Why have breast cancer mortality rates declined? J Cancer Policy [Internet]. 2015;5:817. Available from: http://dx.doi. org/10.1016/j.jcpo.2015.03.002.

8. Lee TS, Kilbreath ÆSL, Refshauge KM, Herbert ÆRD, Beith JM. Prognosis of the upper limb following surgery and radiation for breast cancer. 2008;110:1937. Epub 2007 Sep 26.

9. Schmitz KH, Speck RM, Rye SA, DiSipio T, Hayes SC. Prevalence of breast cancer treatment sequelae over 6 years of follow-up: The pulling through study. Cancer. 2012;118(Suppl.8):2217-25.

10. Bergmann A, Ribeiro MJP, Pedrosa E, Nogueira EA, Oliveira ACG. Fisioterapia em mastología oncológica: rotinas do Hospital do Câncer III/INCA. Bras Cancerol. 2006;52:97-109.

11. Ramírez K, Acevedo F, Herrera ME, Ibáñez C, Sánchez C. Physical activity and breast cancer. Rev Med Chil. 2017;145:75-84.

12. Hidding JT, Beurskens CHG, Van Der Wees PJ, Van Laarhoven HWM, Nijhuis-van Der Sanden MWG. Treatment related impairments in arm and shoulder in patients with breast cancer: A systematic review. PLoS One 2014;9:e96748.

13. Land SR, Kopec JA, Julian TB, Brown AM, Anderson SJ, Krag DN, et al. Patient-reported outcomes in sentinel node-negative adjuvant breast cancer patients receiving sentinel-node biopsy or axillary dissection: National Surgical Adjuvant Breast and Bowel Project phase III protocol B-32. J Clin Oncol. 2010;28:3929-36.

14. Gerber LH, Stout NL, Schmitz KH, Stricker CT. Integrating a prospective surveillance model for rehabilitation into breast cancer survivorship care. Cancer. 2012;118(Suppl.8):2201-6.

15. De Groef A, Van Kampen M, Dieltjens E, Christiaens MR, Neven P, Geraerts I, et al. Effectiveness of postoperative physical therapy for upper-limb impairments after breast cancer treatment: A systematic review. Arch Phys Med Rehabil. 2015;96:1140-53.

16. Rafn BS, Hung S, Hoens AM, McNeely ML, Singh CA, Kwan W, et al.

Prospective surveillance and targeted physiotherapy for arm morbidity after breast cancer surgery: a pilot randomized controlled trial. Clin Rehabil [Internet]. 2018;269215518757292. Available from: http://www.ncbi.nlm.nih.gov/ pubmed/29473482

17. Ramírez-Parada K, Barra-Navarro M, Henríquez-Correa C, Méndez-Zedan P, Vallejos-Aburto P. Factibilidad de implementar un programa de ejercicio físico precirugía en mujeres con cáncer de mama. Tesis de pregrado para optar al título de Licenciatura en Kinesiología. Carrera de Kinesiología. Facultad de Medicina. Pontificia Universidad Católica de Chile. 2018.

18. Cheville AL, Nyman JA, Pruthi S, Basford JR. Cost considerations regarding the prospective surveillance model for breast cancer survivors. Cancer. 2012;118(Suppl.8):2325-30.

19. Binkley JM, Pusic AL, Soballe PW, McNeely ML, Campbell KL, Gabram S. A prospective model of care for breast cancer rehabilitation: Postoperative and postreconstructive issues. Cancer 2012;118(S8):2226-36.

20. Stout NL, Binkley JM, Schmitz KH, Andrews K, Hayes SC, Campbell $\mathrm{KL}$, et al. A prospective surveillance model for rehabilitation for women with breast cancer. Cancer 2012;118(Suppl.8):2191-200. 
CIRUGía AL díA

21. Stubblefield MD, Keole N. Upper Body Pain and Functional Disorders in Patients With Breast Cancer. PMR 2014;6:170-83.

22. Moskovitz AH, Anderson BO, Yeung RS, Byrd DR, Lawton TJ, Moe RE. Axillary web syndrome after axillary dissection. Am J Surg. 2001;181:434-9.

23. Yeung WM, McPhail SM, Kuys SS. A systematic review of axillary web syndrome (AWS). Vol. 9, Journal of Cancer Survivorship 2015;9:576-98.
24. Jiménez Tordoya EJ. Guía metodológica para elaborar el diagnóstico fisioterapéutico según la Clasificación Internacional del Funcionamiento (CIF), de la discapacidad y de la salud. Gac Médica Boliv. 2016;39:46-52. 